\title{
TEES: A Novel Multiple Criteria Optimization Scheme for Temperature-constrained Energy Efficient Storage
}

\author{
Jian Zhou ${ }^{12}$, Jun Wang ${ }^{2}{ }^{*}$, Fei $\mathrm{Wu}^{1 *}$, and Changsheng Xie ${ }^{1}$ \\ ${ }^{1}$ Wuhan National Laboratory for Optoelectronics, \\ Huazhong University of Science \& Technology \\ ${ }^{2}$ Department of Electrical Engineering and Computer Science, \\ University of Central Florida \\ *Corresponding Author: jwang@eecs.ucf.edu,wufei@hust.edu.cn
}

\begin{abstract}
Existing energy saving schemes that have been developed for Energy Efficient Storage funnel I/O traffic on a few disks while allowing the rest idle. These schemes can cause long standing disks to overburden, resulting in a higher rate of disk failure and reliability degradation. In this paper, we develop a novel multiple criteria optimization scheme based on Fuzzy Decision Making theory, for the Temperature-constrained Energy Efficient Storage System called TEES. TEES aims to enforce a temperature constraint as well as performance requirements while also keeping energy consumption to a minimum. This is achieved by developing an online temperature prediction model and aggregating all the decision criteria, such as I/O performance, power consumption, estimated temperature and frequency of disk-status transition. The experimental results show that TEES is able to reduce disk temperature by 20 $30 \%$ as compared with existing control methods, while obtaining comparable performance and power consumption.
\end{abstract}

Keywords : Temperature-constrained, Fuzzy control, Energy-efficient storage.

\section{Introduction}

Due to staggering electricity bills and high disk failure rates, an increasingly large number of energy and temperature control requirements have been introduced on modern storage systems. In order to meet these requirements, data centers have been considering increasing the setpoint temperature at which to run the cooling system[1], [2]. On the other hand, various energy conservation schemes in storage systems have been developed to aggregate heavy workloads on a few disks with a method called Energy Efficient Storage. While both of these methods reduce energy consumption, they could elevate temperature levels on long standing disks and ultimately cause them to overheat. S. Yin [3] studied the reliability model of Energy Efficient Storage, which indicated that the temperature between disks, the age of the disks, and the distribution of workload between disks can elevate their failure rate. N. El-Sayed [2] found that of the three factors listed above, the temperature of the disks had the greatest influence on their failure rate.

Researchers have developed several well recognized temperature constrained schedule schemes for CPUs[4], clusters[5], and connection intensive applications[6]. J. Moore [7] and C. Bash [8] developed open-loop search and optimization methods based on the assumption that the power consumption of chip multiprocessors (CMPs) at each Dynamic Voltage and Frequency Scaling (DVFS) level can be estimated accurately. While this method is effective when the workload pattern is predictable, dramatic workload changes may lead to severe performance degradation or even power constraint violations.

Recently, several closed-loop control solutions have been developed for CMPs using heuristics [4], [5], that employ basic control theory such as model predictive control (MPC). However, MPC requires a predefined fixed temperature constraints for the controlled system[9]. The fixed temperature constraint is used to initiate the process of cooling down the disks if their temperature surpasses a certain limit. This rigid control has several drawbacks. First, the optimal temperature at which to set constrains can often vary in real world applications. Also, if the workload rises to an extreme level, the MPC will be unable to find a low temperature working groups at which the desired constraint can be achieved without using mixed constraints or multi-objective functions. If the constraint is set at a high temperature, the disks will be in danger of failure. If we set multiple levels of constraints, the system may still be exposed in frequently disk status switching when performing the transfer between each level. The Fuzzy Decision Making implemented by 
TEES can allow for a smooth transition between a range of constraints, thus eliminating the possibility of a sudden spike in the storage system. Second, multiple constraints such as warrantable service time, power-state transition frequency of given disks, etc. [3], [10] need to be formalized in the predefined model. This makes the design of a MPC controller very complex. TEES can simplify multidimensional optimization into one dimensional optimization by aggregating all the criteria.

In this paper, we attempt to apply fuzzy control theory to thermal management in order to reduce disk temperature in energy-efficient storage systems. Compared to the existing work, the following contributions has been made.

- We made the first attempt to address the temperature overhead in existing Energy Efficient Storage.

- We employed Fuzzy Decision Making to achieve complexity multidimensional optimization.

- We designed a precise temperature predict model for disks which granted the control accuracy.

- We simulated the large scale Temperatureconstrained Energy Efficient Storage to prove the scalability of our control method.

The paper is organized as follows. Section II describes our motivation. Section III describes the modeling, design and analysis of Fuzzy Decision Making functions. Section IV provides the implementation details and Section $\mathrm{V}$ presents extensive experiments and results. Finally, Section VI concludes the paper.

\section{Motivation \& Background}

\section{A. Energy Efficient Storage}

Parallel disk storage systems, such as RAID, disperse the workload evenly between all disks in order to maximize their performance. However, these storage systems also maximize their power consumption. As the scale of the storage system increases, the power consumption becomes a critical factor. Various methods regarding the power conservation of storage systems have been implemented in recent years. The most rudimentary method, known as Energy Efficient Storage, utilizes a few disks to perform a given task while keeping the remaining disks powered down. We categorize current implementations of energy-efficient storage into cachebased and gear-based.

Cache-based energy-efficiency storage[11], [12], [13], [14], [15], [16], [17], [18], adds some additional storage devices as cache. Unfortunately, the cache-based energy-efficiency storage assumes that the I/O workload has a predictable time locality and good spatial locality and that the load intensity is small.
Gear-based energy-efficient storage[19], [20], [10], [21], [22] is designed to always guarantee data availability by providing multiple replicas of the data. Figure 1 illustrates a minimal implementation of Power Proportional Storage (Rabbit [22]) on 16 disks with 3 replicas. Group 1 has 3 disks that contain the first replica. Group2 has 5 disks that contain the second replica. Group3 has 8 disks that contain the third replica. When the system I/O workload is low, only Group 1 is serving the request, Group2 and Group3 is powered off to save energy. When the system I/O workload becomes high, disks from Group 2 and Group3 are powered up one by one to meet the performance requirements.

Unfortunately, this scheduling scheme sacrifices reliability by overburdening some disks while ignoring others. All the disks in Group 1 will always work under heavy workloads, this can cause those disks to overheat. High temperature is the main factor that increases the disks' latent sector error rate and failure rate[2]. In our experiment, the disks in Group 1 can reach temperatures of up to $50^{\circ} \mathrm{C}$ in thirty minutes under heavy workloads with a normal environment temperature (around $28^{\circ} \mathrm{C}$ ), which is very dangerous[2].

\section{B. Temperature Overheat}

Several studies have been conducted for the purpose of studying the relationship between disk temperature and failure rate. A study conducted by Google in 2007 [23] suggested that lower temperatures are actually more detrimental to disk reliability than higher temperatures. However a research conducted by Microsoft in 2011 [24], [25] demonstrated that Annualized Failure Rate (AFR) steadily increases as Hard Disk Drive (HDD) temperature increases. The most recent research conducted by N. El-Sayed in 2012 [2] suggests that the reason why Google's study arrived at the conclusion is because when the study was conducted, many disks of various models were used to aggregate the data and different models of disks failed at different temperatures. N. El-Sayed [2] observed that the increase in failures with respect to temperature tends to be linear, except for very high temperatures (above $50^{\circ} \mathrm{C}$ ). Because of this relationship between disk temperature and failure [2], [24], [25], [26], a temperature constrained control scheme is essential to the well-being of Energy Efficient Storage systems.

\section{Thermal-constrained Scheduling}

We can design a temperature-constrained scheduling for energy-efficiency storage by switching the workload to idle disks when the temperature reaches a certain threshold. For example, when the workload is below $18 \%$ of the full performance of the storage in Figure 
1 , the storage system can serve the I/O requests with any of the disk groups. The scheduler may be able to switch the workload between groups to bring the disks' temperature under a safe threshold, e.g. $36^{\circ} \mathrm{C}$.

However, this policy is not going to work in the real word. If the workload intensity increases, the scheduler will require more disks to serve the $\mathrm{I} / \mathrm{O}$ requests, and hence fewer idle disks can be selected to replace a given disk that is overheating. This implies that a overheated disk will have less time to cool down before it is reinstated as a working disk; therefore, the status of a given disk will be switched more frequently. Frequently powering up and down a disk will greatly reduce its lifetime [10]. Moreover, the status switching is a time consuming process because of the lazy-update introduced in energy-efficient storage[27]. When a disk is powered down, the updated data on that disk must first be transferred to the disk that will replace it. If the temperature of the candidate group reaches the threshold before the switch is completed, the system will out of control.

A straightforward solution could be to implement rule based fuzzy control, which sets multiple temperature thresholds. This would imply that whenever a threshold is reached, the status of some or all of the disks will be switched more often. This frequent switching of status' can be very detrimental to the overall lifespan and performance of the disks.

Normally, the power consumption of an energy efficient storage system is generally proportional to its performance. However, when a temperature-constrained scheduler is introduced, more disks, and thus more power, can be needed to serve the same I/O load. This is because a temperature-constrained scheduler must balance power consumption, temperature, and several other parameters as well as performance. Achieving this balance can be a complicated task. For example, we know that introducing temperature constraints can elevate the power consumption. However, we can not find a direct relationship between temperature and power consumption, because their relationship also can be influenced by performance.

Because of this complexity, we have chosen temperature as the focus of this paper. If another factor must be taken into account, e.g. disk utilization or electricity bill [28] our model can be expanded by integrating this new factor, along with other reliability-affecting factors, in TEES.

Moorey et. al. developed three dynamic thermal managements [7] to deal with the stability of the scheduling arithmetic, avoid the changes in temperature and minimize the cost of thermal management. Heath et. al. developed a thermal emulation model called Mercury along with a very simple thermal feedback control called Freon [29]. These arithmetics simply schedule the load to the low temperature node, but may schedule the load to a machine that is hard to cool. In our design, an online temperature estimation model is used to avoid this. Abbasi proposed a twotier dynamic server provisioning and workload distribution method in developing thermal aware Internet data centers[30]. Weissel et. al. used Newton's Law of Cooling to predict dynamic thermal management for distributed systems[31]. Ramos et. al. improved the CFreon in C-Oracle[32] using online thermal prediction. Their basic method is to control the load intensity of a server. Wang et. al. introduced the online model to predict the CPU temperature control [4]. However, they do not study at which temperature should we set the constraints. Our design gives a flexible constraint for the temperature while balancing it with multiple other constraints.

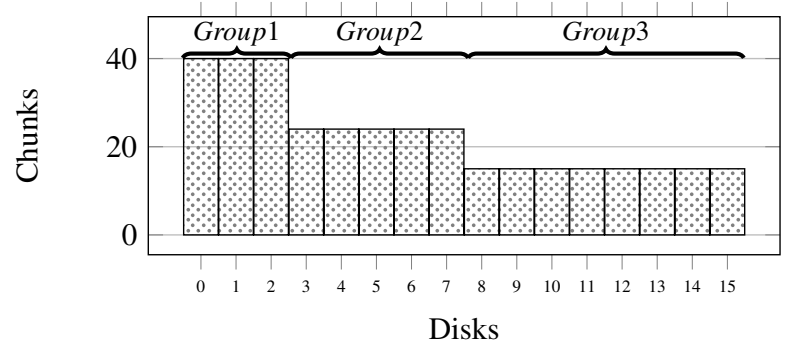

Fig. 1. Power-Proportional Layout.

\section{Design and Methodology of TEES}

In order to perform temperature-constrained control, we designed a feedback control loop. As shown in Figure 2, the key components in the control loop include a temperature sensor on each disk, a power monitor connected to the power supply circuit of each disk and an online model estimator. Let $S P$ denote the sampling period; and $k$ denote the sampling point. $S_{k}$ denotes current time active disk set at time $k$ including the number of disks used and how they are combined or organized for the replicas; $L$ denotes the data layout referring how the data blocks are distributed on the disks and in this paper we only focus on the powerproportional layout mentioned above; $\operatorname{Per} f_{k}$ denotes the required performance by the user at time $k ; P_{k}$ denotes the are measured disk power consumption; $T_{k}$ denotes the measured disk temperature; and $O_{k}$ denotes the operation time since the last spin down or spin up a disk. $S_{k}, L, P e r f_{k}, P_{k}, T_{k}$ and $O_{k}$ are all input signals for our fuzzy controller to calculate an appropriate active disk set in the next time window SP. We express our feedback control loop in the following function: 


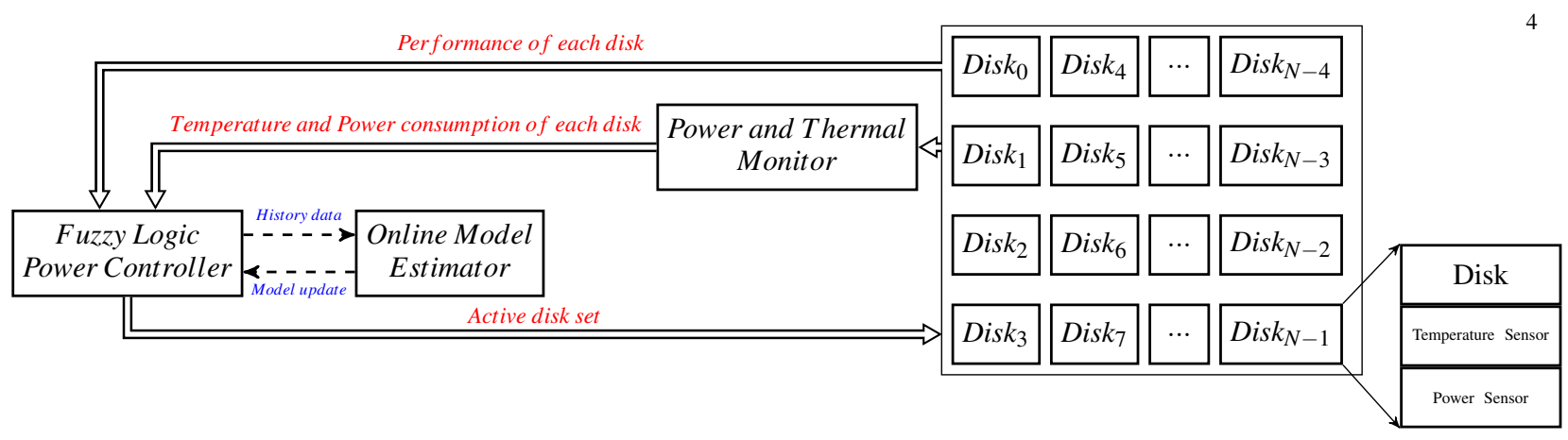

Fig. 2. Temperature-constrained energy control loop for an Energy Efficient Storage system with N disks.

$$
S_{k+1}=F\left(S_{k}, L, \operatorname{Perf}_{k}, P_{k}, T_{k}, O_{k}\right)
$$

where, $F$ denotes the feedback algorithm.

The control loop is invoked periodically and its period is chosen based on a trade-off between actuation overhead and system settling time. The following steps are invoked at the end of every control period. 1) The monitors collect the temperature and power consumption of each disk as well as the overall performance status of the storage system. 2) The online model estimator updates its parameter based on the collected data. 3) Based on the collected data, the controller computes future temperatures of the storage system and selects the working disks accordingly.

We propose Fuzzy Decision Making (FDM) as the feedback algorithm $F$ [33] for the temperatureconstrained scheduler for Energy Efficient Storage. This method defines a range of temperature constraints and quantifies the constraints with a satisfaction degree from 0 to 1 ; thus, a smooth control can be achieved.

The controller uses the system model to predict the control behavior over sampling periods, $H_{p}$. This is called the prediction horizon. The control objective is to select an input trajectory that minimizes the objective function. The objective function is formulated to represent the satisfaction of the decision criteria and control goals after applying the control actions in the entire prediction horizon, $H_{p}$. An input trajectory includes the control inputs in the following $H_{m}$ sampling periods. $S_{k+1}, \ldots, S_{k+H_{m}}$, where $H_{m}$ is called the control horizon. The notation $S_{k+i}$ means that the disk state vector of the storage system at time $k+i$ depends on the conditions at time $k$. Once the input trajectory is computed, only the first element $S_{k+1}$ is applied as the control input to the system. At the end of the next sampling period, the prediction horizon slides on sampling period and the input is computed again based on the feedback from the performance, power and temperature monitors.

\section{A. Multi-criteria Constraints in Power Consumption Optimization}
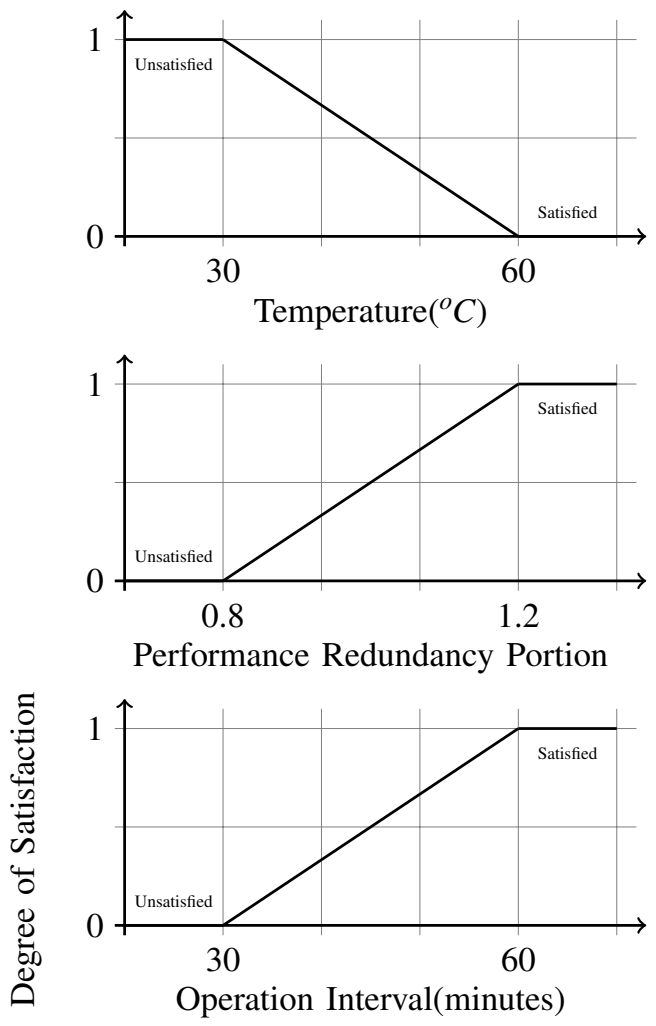

Fig. 3. Liner Satisfaction Membership

Because I/O performance, Power Consumption, Temperature, and Operation Intervalare measured with separate continuous units, their individual satisfaction levels can be mapped to the interval $[0,1]$. The control object is subjected to three sets of constraints. First, the performance level should meet the minimal requirements of the workload. Second, the temperature of each disk should be below a given threshold (e.g., $50^{\circ} \mathrm{C}$ ). Third, the interval between power up and shut down of a certain disk should be above a given time (e.g., 30 


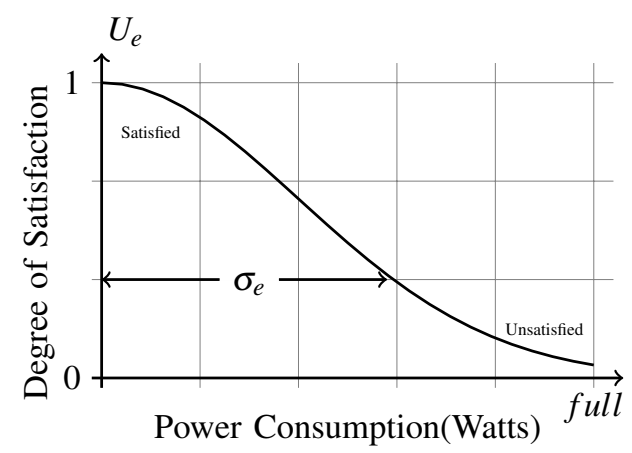

Fig. 4. Exponential Satisfaction Membership

minutes). In MPC, these constraints are the "hard" constraints, which could create a scenario in which no optimal solution can be achieved [34]. Therefore, in our design, fuzzy constraints are used. For simplicity and reserving the "hard" constraints to indicate the physical limitation, the satisfaction of the fuzzy constrains is expressed by the liner membership function shown in Figure 3.

The fuzzy temperature constraint criteria indicate how well the temperature of each disk is satisfied. Basically, there are two types of temperatures regarding the disk failure model. Sankar states that there is an exponential relationship between the temperature and disk failure models [24]. El-Sayed points out that for temperatures below $50^{\circ} \mathrm{C}$, disk failure rates grow as a linear function [2] when the temperature increases. Both of the models can be used as the temperature membership function. For simplicity, the fuzzy temperature constraint is formulated by the following equation:

$$
\mu_{t(k+i)}=\left\{\begin{array}{l}
1, T_{k+i} \leq \sigma_{t 0} \\
\frac{\sigma_{t 1}-T_{k+i}, \sigma_{t 0}<T_{k+i}<\sigma_{t 1}}{\sigma_{t 1}-\sigma_{t 0}} \\
0, T_{k+i} \geq \sigma_{t 1}
\end{array}\right.
$$

Where, $\sigma_{t 0}$ and $\sigma_{t 1}$ are the temperature at which the constraint is fully satisfied and unsatisfied respectively. For a regular storage system, $\sigma_{t 0}$ could be $30^{\circ} \mathrm{C}$, and $\sigma_{t 1}$ could be $55^{\circ} \mathrm{C}$.

The fuzzy performance constraints criteria indicate how well the performance requirements are satisfied. Sufficient performance redundancy are essential for storage systems, in order to ensure successful and reliable data service. The fuzzy performance constraints can be formulated by the following equation:

$$
\mu_{p(k+i)}=\left\{\begin{array}{l}
0, \frac{\operatorname{Perf}_{k+i}}{\operatorname{Perf}_{k}} \leq \sigma_{p 0} \\
\frac{\operatorname{Perf}_{k+i}}{\operatorname{Perf}_{k}-\sigma_{p 0}}, \sigma_{p 0}<\frac{\operatorname{Perf} f_{k+i}}{\operatorname{Perf}_{k}}<\sigma_{p 1} \\
1, \frac{\sigma_{p 1}-\sigma_{p 0}}{\operatorname{Perf}_{k}} \geq \sigma_{p 1}
\end{array}\right.
$$

Where, $\sigma_{p 0}$ and $\sigma_{p 1}$ are the portions of performance redundancy at which the performance constraints are fully unsatisfied and satisfied respectively. These constraints could be selected based on specific workloads. For a regular storage system, $\sigma_{p 0}$ could be 0.8 , and $\sigma_{p 1}$ could be 1.2 .

The fuzzy operation interval constraint criteria indicate the time interval between two disks status switching. It is formulated by the following equation:

$$
\mu_{O(k+i)}=\left\{\begin{array}{l}
0, O_{k+i} \leq \sigma_{o 0} \\
\frac{O_{k+i}-\sigma_{o 0}}{\sigma_{o 1}-\sigma_{o 0}}, \sigma_{o 0}<O_{k+i}<\sigma_{o 1} \\
1, O_{k+i} \geq \sigma_{o 1}
\end{array}\right.
$$

Where, $\sigma_{o 0}$ and $\sigma_{o 1}$ are the interval at which the constraints fully unsatisfied and satisfied respectively. For a regular storage system, $\sigma_{o 0}$ could be 30 minutes, and $\sigma_{o 1}$ could be 60 minutes.

A fuzzy set in the appropriate domain characterizes both the fuzzy goals, such as reducing the power consumption, and the fuzzy constraints, such as temperature constraints. Applying FDM in the temperatureconstrained power controller allows the combination of goals and constraints to be achieved. The estimated power consumption is mapped to the interval $[0,1]$ which indicates how well the power consumption satisfies the goal to minimize it by utilizing a membership function. The fuzzy goals are defined by a Gaussian membership function that is never been zero, as shown in Figure 4. That indicates that high power consumption is an allowable but not a desirable state. The fuzzy goal can be formulated by the following equation:

$$
\mu_{e(k+i)}=\exp \left(-\frac{\left(S_{k+i} P_{k}\right)^{2}}{2 \sigma_{e}^{2}}\right)
$$

Where $S_{k+i}$ is the disk state vector at time $k+i, P_{k}$ is the power consumption vector of each disk, $\sigma_{e}$ is used to determine how fast the fuzzy goal approaches as the power consumption increase. For a regular storage system, the $\sigma_{e}$ could be half of the max energy consumption in a sampling period.

\section{B. Aggregation of Criteria for Energy Efficient Storage}

Because all of performance criteria can affect each other, the satisfaction levels of all of these factors must be aggregated into an equation and maximized. The fuzzy criteria aggregation is the process that computes the joint satisfaction of all the criteria [34]. The confluence of goals and constraints can be done by aggregating the membership values. The membership value $\mu_{\pi}(k)$ for the control sequence $\pi$ is obtained using the aggregation operators $\otimes, \otimes_{g}$ and $\otimes_{c}$ to combine the decision criteria in Equation 6: 


$$
\begin{aligned}
& \mu_{\pi}(k)=\left(\mu_{e(k+1)} \otimes_{g} \ldots \otimes_{g} \mu_{e\left(k+H_{p}\right)}\right) \\
& \otimes\left(\mu_{p(k+1)} \otimes_{c} \ldots \otimes_{c} \mu_{p\left(k+H_{p}\right)}\right) \\
& \otimes\left(\mu_{t(k+1)} \otimes_{c} \ldots \otimes_{c} \mu_{t\left(k+H_{p}\right)}\right) \\
& \otimes\left(\mu_{o(k+1)} \otimes_{c} \ldots \otimes_{c} \mu_{o\left(k+H_{p}\right)}\right)
\end{aligned}
$$

In Equation $6, \otimes_{g}$ denotes an aggregation operator for combining the goals, $\otimes_{c}$ denotes an aggregation operator for combining the constraints, $\otimes$ denotes an aggregation operator for combining the aggregated goals and constraints. The operator $\otimes_{g}$ is the average operator. It computes the average satisfaction of the energy consumption. So, the accumulated energy consumption, rather than the energy consumed in certain sampling period, is taken into account. The operator $\otimes_{c}$ is the minimum operator. The smallest satisfaction of the constraints are chosen as the decision criteria. The operator $\otimes$ is the weighted averaging operator.

The control object is to find a solution that best satisfies the joint constraint of all the fuzzy criteria. The ideal results in which we desire is a situation that all the criteria are satisfied. In this case, the storage system will get 1) sufficient performance redundancy, 2) low temperature of disks, and 3) few disks spin down spin up actions, and 4) minimal energy consumption in the prediction horizon. The requirement manifested by an "and" operator of the criteria values. The ordered weighted averaging aggregation operators [35] are used in the aggregation of goals and constraints. Assume the ordered weight vector $W$ is $\left[w_{e}, w_{p}, w_{t}, w_{o}\right]$, the $\mu_{\pi}$ can be expressed as:

$$
\mu_{\pi}=w_{e} \mu_{e}+w_{p} \mu_{p}+w_{t} \mu_{t}+w_{o} \mu_{o}
$$

Where $w_{e}+w_{p}+w_{t}+w_{o}=1$.

The translation of control goals and constraints to a membership value avoids the specification of the criteria in a large multidimensional space. The control function $F$ in Equation 1 then can be expressed as finding active disk group $S_{k+1}$ that satisfy the decision criteria $\mu_{\pi}$ as much as possible, which corresponds to the maximal value of the overall satisfaction. Thus, the optimal sequence of control actions $\pi^{*}$ is found by the maximization of $\mu_{\pi}$.

$$
\pi^{*}=\operatorname{argmax}\left(\mu_{\pi}\right)
$$

\section{Temperature Prediction}

In the previous subsection, we defined the control function which can be used to make decision of choosing the active disk sets. However, in practice the control decision needs to be made based on a list of future criteria. This is mainly because the lazy-update feature of disk temperature mentioned in Section II requires that the scheduler switches the status of a disk that is about to overheat before the temperature actually reaches the threshold. Therefore the controller needs to predict the future temperature then use the decision criteria in Equation 8 to guarantee qualitative control results. To predict the disk temperature we build a thermal model based on several classic energy theories. The conservation of energy represents the most basic equation in heat transfer. There are two sources of heat dissipation on a disk: heat produced by the hard drive when disk is working, and heat that is gained or lost from the environment. We first introduce some notations. Suppose our sampling interval is $\hat{t}$, and $i(1 \leq$ $i \leq N)$ is one of the sample points. The heat variation of a certain disk at time $i$ during time span $\hat{t}$ can be expressed as:

$$
Q_{\text {gain }, i}=Q_{\text {produce }, i}+Q_{\text {transfer }, i}
$$

Where $Q_{\text {gain }, i}$ denotes the amount of heat that the disk gained during time $\hat{t}, Q_{\text {produce }, i}$ denotes the amount of heat produced by the performing work during time $\hat{t}$, and $Q_{\text {transfer, } i}$ denotes the amount of heat transferred from/to the disk during time $\hat{t}$.

The transport equations for thermal energy (Fourier's law), mechanical momentum (Newton's law for fluids) and mass transfer (Fick's laws of diffusion) are similar. Analogies among three transport processes have been developed to facilitate prediction of conversion from anyone to others. The heat transferred from/to the environment can expressed as:

$$
Q_{\text {transfer }, i}=Q \cdot\left(T_{i}-T_{0}\right) \cdot S P
$$

Where $Q_{\text {transfer, } i}$ is the amount of heat transferred between a disk and its environment during time $\hat{t}, Q$ is a constant that embodies the heat transfer coefficient and the surface area of the object, $T_{i}$ is the temperature at sampling point $i, T_{0}$ is the temperature of the environment, $S P$ is the sampling period.

The heat produced by a component essentially corresponds to the energy it consumes, so we define it as:

$$
Q_{\text {produce }, i}=P_{i} \cdot S P
$$

Where, $p_{i}$ is the power factor of disk at time $i$.

Since pressures and volumes at the disk are assumed constant, the temperature is directly proportional to its internal energy. More formally, we define the object's temperature variation as:

$$
Q_{\text {gain }, i}=C \cdot\left(T_{i+1}-T_{i}\right) \cdot S P
$$


Where $\mathrm{C}$ is the constant that embodies the mass of the object and its specific heat capacity.

Referring to the above model, we summarize the temperature model as the following formula:

$$
T_{i+1}-T_{i}=C_{1} \cdot\left(T_{i}-T_{0}\right)+C_{2} \cdot P_{i}
$$

Where $C_{1}$ is $Q / C, C_{2}$ is $1 / C$.

\section{Online Model Update}

In this section, we discuss how to perform the online model update based on the history data. The online model update refers the process of calculating arguments used in the temperature estimation model. We use recursive least square (RLS) to calibrate a constant $C_{1}$ and $C_{2}$ which best match the history. Let's simplify the thermal model in Equation 13 as follows:

$$
Y_{t+1}=C_{1} \cdot \hat{T}_{t}+C_{2} \cdot P_{t}
$$

Where, $Y_{t}$ is $T_{t+1}-T_{t}, \hat{T}_{t}$ is $T_{t}-T_{0}, P_{t}$ is disk power consumption.

Then we get the weighted squared error:

$$
\left.\chi_{i}=\sum_{i=1}^{m} \lambda^{m-i}\left(Y_{i}-C_{1}(i) \cdot \hat{T}_{i}-C_{2} \cdot P_{i}\right)\right)^{2}
$$

Where, $0<\lambda<1$ is the "forgetting factor" which gives exponentially less weight to older error samples, and $m$ is the total number of history data used in the model update. A smaller $\lambda$ allows the estimator to forget the history data more quickly. In our experiments, we use $\lambda=0.8$.

The squared error is minimized by taking the partial derivatives for all entries $C_{1}$ and $C_{2}$ of the coefficient vector $\chi$ and setting the results to zero.

$$
\left\{\begin{array}{l}
C_{1} \cdot \sum T_{i}+C_{2} \cdot \sum\left(P_{i} \cdot T_{i}\right)=\sum\left(Y_{i} \cdot T_{i}\right) \\
C_{2} \cdot \sum P_{i}+C_{1} \cdot \sum\left(P_{i} \cdot T_{i}\right)=\sum\left(P_{i} \cdot Y_{i}\right)
\end{array}\right.
$$

Where, $\Sigma=\sum_{i=1}^{m} \lambda^{m-i}$. And then,

$$
\begin{gathered}
C_{1}=\frac{\sum\left(Y_{i} \cdot T_{i}\right) \sum P_{i}-\sum\left(P_{i} \cdot Y_{i}\right) \sum\left(P_{i} \cdot T_{i}\right)}{\sum T_{i} \sum P_{i}-\left(\sum\left(P_{i} \cdot T_{i}\right)\right)^{2}} \\
C_{2}=\frac{\sum\left(P_{i} \cdot Y_{i}\right) \sum T_{i}-\sum\left(Y_{i} \cdot T_{i}\right) \sum P_{i}}{\sum T_{i} \sum P_{i}-\left(\sum\left(P_{i} \cdot T_{i}\right)\right)^{2}}
\end{gathered}
$$

After we calibrate $C_{1}$ and $C_{2}$, we can estimate the temperature by the Equation 13.

The following iteration is invoked in every control period: 1) The RLS estimator records the power consumption and temperature of each disk; 2) The estimator calibrates $C_{1}$ and $C_{2}$ in the thermal model; 3) The model estimator estimates the temperature in the future. Because the $\left(T_{t}-T_{t-1}\right)$ will converge at 0 , we can predict the equilibrium temperature using Equation 13.

\section{TEES Prototype Implementation}

In this section, we describe our physical experimental testbed and benchmarks, as well as the implementation details of each component in TEES control loop.

We develop TEES prototype on a machine with an Intel Dual-Core E5200 2.5GHz CPU, 2G Bytes DDR2 memory running an Ubuntu 11.04 operating system. We implement a trace replayer on our power-proportional data layout based storage system testbed and measure the performance and power consumption of disks for each run. Due to the hardware limit, the prototype contains 16 disks, which are connected by an SAS cable. To measure the power consumption of disks, we adopt a plug-in real-time multi-meter, called ZH102.

\section{A. Trace Replay Framework}

In our prototype system, we implement the trace replay framework as a $\mathrm{C}$ program running under Ubuntu Server 12.04. Because of the heavy integration of our control algorithm, a new trace replay code is developed rather than using an existing one. It has $8.5 k$ code lines in all. The main idea is to use the "libaio" programming library to asynchronously access $\mathrm{I} / \mathrm{O}$ of the storage subsystem (sending I/O requests to the storage subsystems). The trace replay framework consists of 6 modules: performance requirements monitor, power \& temperature monitor, model predictor, fuzzy controller, data layout manager, and virtual disk layer.

\section{B. Power Proportional Layout}

We implemented power proportional layout [22] in our storage system. In the experiment, we made three replicas of the whole data set in the virtual disk. The first replica is spread on three disks, which are called disk Group 1 in the experiment. The second replica is stored on five disks, which are called disk Group 2 in the experiment. The last replica is saved on eight disks, which are called disk Group 3 in the experiment. We use a total of 16 disks in the experiments.

\section{Write Off-loading}

TEES uses an alternated version of Write Off-loading [27] technique to maintain the power proportional feature. Write Off-loading is an energy saving technique for storage system, which prolongs the idle time of standby disks by redirecting writes to active disks temporarily. It redirects the writes to the currently active disks and updates the writes to the other replicas when a specific trigger occurs. While the trigger used in the original Write Off-loading is the off-loaded time span or data amount, the only trigger used in our experiment, including TEES and other baselines, is that the controller changes the active disk sets based on the constraints. 


\section{Experimental Results and Analyses}

We present our experimental results and associate indepth analyses here.

\section{A. Workload}

We use a mixture of real-world and synthetic traces to comprehensibly study the impact of different storage architectures on a wide spectrum of enterprise-scale workloads. Table I presents salient features of our workloads. We employ a write-dominant I/O trace from an OLTP application running at a financial institution and a popular Internet web search machine [36] made available by the Storage Performance Council (SPC), henceforth referred to as the Financial1, Financial2 and Websearch traces. These traces are collaboratively collected by HP and Storage Performance Council. Exchange [37] was collected at the Microsoft Exchange 2007 SP1 server, which is a mail server for 5000 corporate users. MSN [37] was collected at the Microsoft's several Live file servers. Develop [37] was obtained from a file server accessed by more than 3000 users to download various daily builds of Microsoft Visual Studio. Radius [37] was obtained from a RADIUS authentication server that is responsible for worldwide corporate remote access and wireless authentication.

\begin{tabular}{|l|l|l|l|}
\hline Workloads & $\begin{array}{l}\text { Avg.Req.Size } \\
\text { read/write(KB) }\end{array}$ & $\begin{array}{l}\text { Read } \\
(\%)\end{array}$ & $\begin{array}{l}\text { Avg.Req.Arrv. } \\
\text { Time (ms) }\end{array}$ \\
\hline Financial1 & $2.25 / 3.75$ & 23.2 & 8.19 \\
\hline Financial2 & $2.3 / 2.9$ & 82.3 & 11.08 \\
\hline Websearch & $15.15 / 8.6$ & 99.9 & 2.99 \\
\hline Exchange & $15.15 / 14.5$ & 30.8 & 1179 \\
\hline MSN & $9.6 / 11.1$ & 67.2 & 513 \\
\hline Develop & $18.45 / 10.95$ & 88.6 & 1985 \\
\hline Radius & $124.25 / 12.45$ & 17.1 & 9475 \\
\hline
\end{tabular}

ENTERPRISE-SCALE WORKLOAD CHARACTERISTICS.

\section{B. Baselines}

The first baseline system, referred to Rabbit[22], is a power proportional storage. It ensures ideal powerproportionality, by providing multiple gears of storage to work under different performance requirements. All the baselines including TEES use the same data layout in order for a fair comparison.

The second baseline, referred to Simple Feedback Controller (SFC), is a simple temperature feedback control loop. SFC represents a typical feedback solution which uses the real-time per-disk temperature to control the behavior of disks without relying on an online model estimator. We compare our TEES against SFC to show that a well-designed simple temperature feedback controller may still fail to enforce accurate temperature control and thus degrade performance.
The third baseline, referred to Model Predict Control (MPC), is a recent power management solution in CPUs[4]. As we discuss before, the MPC control of CPUs cannot be directly used in storage system. We implement the MPC controller under the framework of SFC control. It shares most part of the SFC controller. A fundamental difference between SFC and MPC controller is that SFC simply uses the moving average temperature of a selected disk by one step, depending on whether the measured temperature is lower or higher than the set point. In contrast, MPC computes a predicted temperature level for each disk according to the temperature prediction model.

\section{Experiment}

To demonstrate the superiority of the Fuzzy Constraint Satisfaction design in TEES, we conducted a series of real word experiment.

1) Stability: This experiment is done under MSN trace, which has a great workload variation from midnight to midnight, to compare the system stability under different controllers. In Figure 5, the MPC Controller simply gives a fixed temperature constraints at each control period. However, this kind of design does not eliminate the controller switch problem and the possible system instability when the workload or environment temperature changes. Figure 6 illustrates the results of TEES. The result shows that TEES can guarantee a system stability by smoothly adjusting the performance, temperature, and power constraints, and avoiding abrupt and crude constraints switch. TEES, which employed Fuzzy Constraint MPC, provides a much more smooth control performance in process operation.

When TEES and MPC are both optimally designed with different parameters (e.g. threshold), they work very well under a fixed condition in which the workload pattern and environment temperature never changes. However, when the workload changes the pre-defined constraints will no longer be acceptable. The MPC controller begins to frequently change the active disk set, and eventually not able to find a feasible solution. This also makes disks vulnerable to fail and introduce high performance penalty and energy cost.

2) Control Accuracy: This experiment is done under WebSearch traces, which have a relative stable workload, to evaluate the control accuracy of different control strategy. The control accuracy is mainly granted by the accuracy of temperature prediction. Based on the prediction, the controller adjusts the workload dispatch policy and transfers the hardware statuses. Otherwise, the control action may be misconducted. While the temperature prediction model is adopted in both TEES and MPC, we randomly select a Web Server trace to test MPC and SFC under a scenario where the temperature 


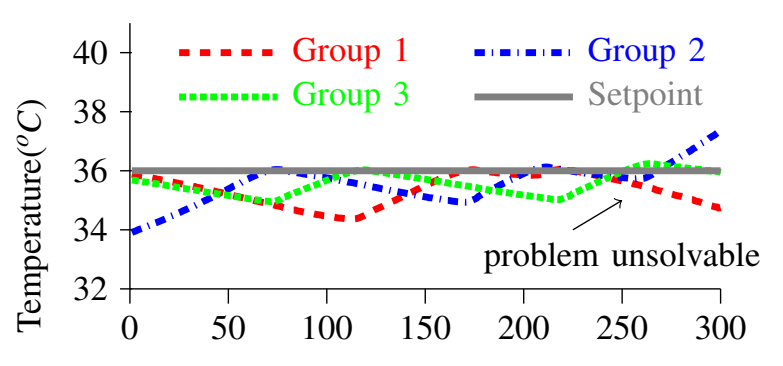

Time(minutes)

Fig. 5. Problem becomes unsolvable for MPC. With fixed temperature constraint the controller can not work properly under different workloads.

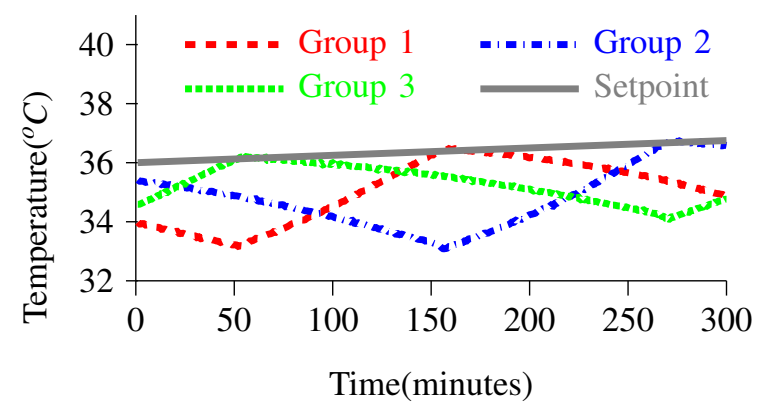

Fig. 6. Soft constraints for TEES. In TEES, the setpoint temperature is adaptively selected based on runtime conditions.

threshold is set to be $36{ }^{\circ} \mathrm{C}$ and the temperature of the environment is a constant.

Figure 7 shows the results of SFC, which do not have a temperature estimator. We can see that SFC does a good job at some points in pulling down the disk temperature. However, it cannot precisely constrict the temperature to be lower than a pre-defined threshold. That is because of the following two reasons. First, SFC takes the control action only after it detects the real-time temperature of the active disk group surpassing the set point. This leads to non-negligible response delays in pulling down the temperature. Second, after the SFC takes the control action, it needs to wait for a preparation delay before the group can be really spun down.

Figure 8 shows that, with an accurate temperature estimator, the controller can precisely constrict the disk temperature under the set point, with a standard deviation smaller than $0.1^{\circ} C$. That is benefited by the accuracy of both the temperature model (detailed in Section III-C) and the online model estimation algorithm (detailed in Section III-D). The online model predictor can precisely estimate the temperature trend of the disks, so that the controller can take control actions before the temperature reaches the threshold.

Figure 9 shows the result of steady-state temperature

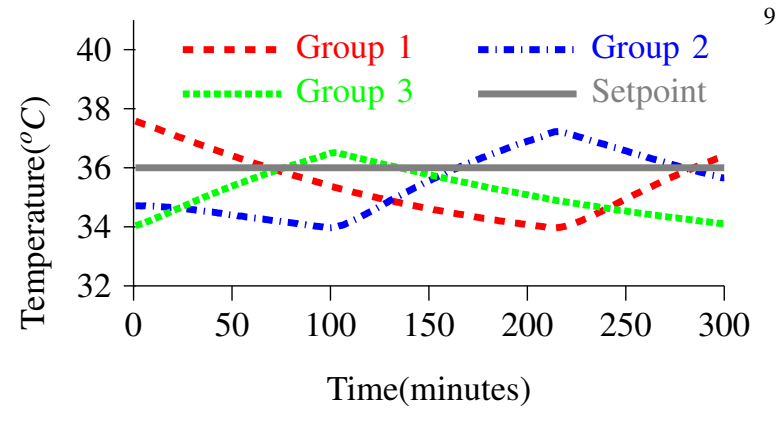

Fig. 7. Control accuracy when temperature estimation disabled. The temperature of active disk set surpass the setpoint between each control interval.

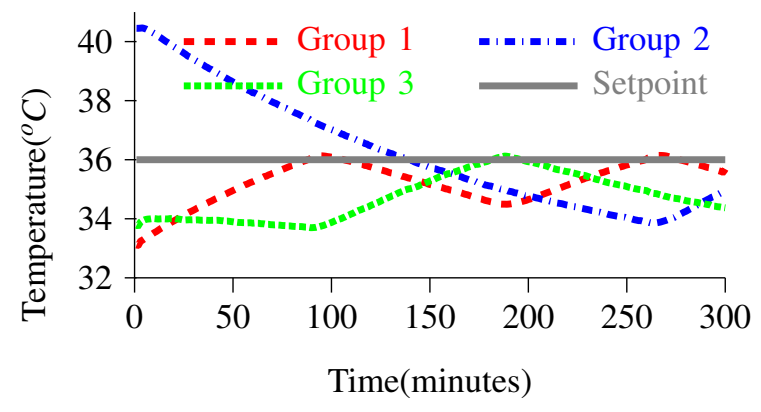

Fig. 8. Control accuracy when temperature estimation enabled. All the disk temperatures are controlled precisely below the setpoint.

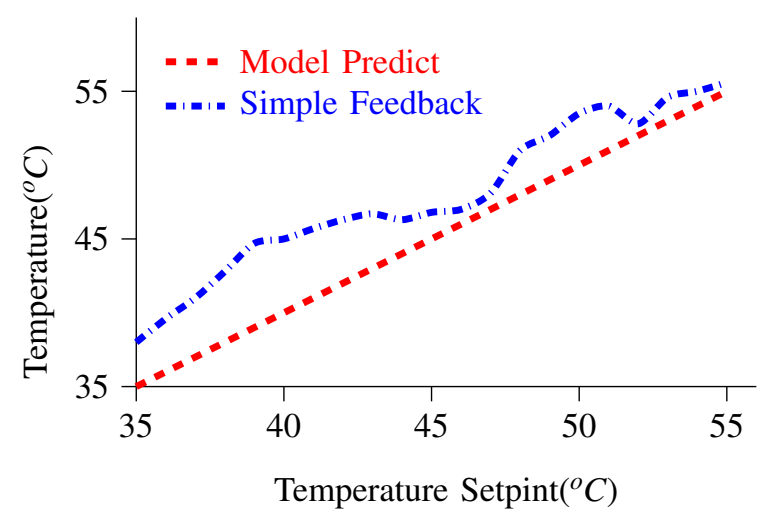

Fig. 9. Steady state error in the real word. TEES use model prediction technique to guarantee a specific level of accuracy in the control.

under a series of temperature set points from $35^{\circ} \mathrm{C}$ to $55^{\circ} \mathrm{C}$. The controller can meet all the set points with a precision less than $1 \%$. However, since the steadystate error occurs mainly because of the delay of control action, the SFC shows a steady state error often above the set point.

3) Multi-Constraints Optimization: One of the main contribution of TEES is that it provides a better way to perform multi-constraints optimization between per- 


\begin{tabular}{|l|l|l|l|l|}
\hline & $\begin{array}{l}\text { Temp. }\left({ }^{\circ} C\right) \\
\text { Min/Avg/Max }\end{array}$ & $\begin{array}{l}\text { Power }(W) \\
\text { Avg }\end{array}$ & Actions & $\begin{array}{l}\text { Satisfy } \\
\text { Avg }\end{array}$ \\
\hline TEES & $33.5 / 38.6 / 39.8$ & 48 & 4 & 0.79 \\
\hline MPC & $36.0 / 38.0 / 38.5$ & 46 & 9 & 0.46 \\
\hline SFC & $30.2 / 40.3 / 42.0$ & 47 & 6 & 0.38 \\
\hline
\end{tabular}

Multi-CONSTRAints Optimization.

\begin{tabular}{|l|l|l|l|l|}
\hline $\begin{array}{l}\text { Replay } \\
\text { Speed }\end{array}$ & $\begin{array}{l}\text { Temp. }\left({ }^{\circ} C\right) \\
\text { Min/Avg/Max }\end{array}$ & $\begin{array}{l}\text { Power }(W) \\
\text { Avg }\end{array}$ & Actions & $\begin{array}{l}\text { Satisfy } \\
\text { Avg }\end{array}$ \\
\hline x4 & $33.5 / 38.6 / 39.8$ & 88 & 4 & 0.72 \\
\hline X8 & $36.0 / 39.2 / 38.5$ & 130 & 5 & 0.63 \\
\hline X16 & $37.9 / 45.3 / 42.0$ & 156 & 8 & 0.48 \\
\hline x32 & $41.2 / 48.3 / 52.0$ & 162 & 10 & 0.35 \\
\hline
\end{tabular}

Multi-Constraints Optimization AcCElERATEd Trace REPLAY.

formance requirement, temperature, power consumption and number of disk status transfer actions.

In this experiment, we carefully choose the parameters for TEES, MPC and SFC, which guarantee that the system can run smoothly in a manipulated environment without any interference on performance. The experiment runs for a fixed 3 hours under WebSearch traces. We get the different running state in Table II. We can see that TEES provides a better way to control aggregated satisfaction of multiple constraints compared to MPC and SFC, since they do not do multiple constraints' optimization and are not able to adjust the constraints automatically. In this table, TEES has fewer total control actions and achieves better aggregated satisfaction degree. For MPC and SFC, aggregated satisfaction degree can be designed to be higher if we configure the system in the right way at each run. However, this is not practical.

In order to study the system behavior under heavy workloads, we accelerated the replay speed while replaying WebSearch traces. In Table III, we can find that the controller relatively consumes more power when the workload is low. This is because of the exponential power consumption satisfaction model has smaller slope at the lower power consumption points. This design can help reserve more system performance and reduce the temperature when the workload is low. When the workload is high, the controller works harder. In this experiments, the system reduce the system I/O performance to maximize the satisfaction degree.

4) Temperature: In this experiment, we evaluate the reduction of overall disk temperature of TEES at different I/O patterns, compared to the power proportional storage - Rabbit [22]. In order to do a fair comparison, we choose looser constrictions for SFC and MPC, so that they are able to run stably at various conditions, because they do not have a well designed constrict change policy. Figure 10 plots the normalized average temperatures of disks with different control methods under different workloads. The result shows that TEES can significantly reduce the temperature adaptively and accurately based on the online model prediction and fuzzy decision making. Figure 11 plots the temperatures of TEES and Rabbit with accelerated trace replay. In this experiment, we want to see what will happen when we get a heavier workload with the same access pattern. Websearch and Financial Traces are randomly selected in the experiment. We steer up higher I/O intensity by multiplying the replay speed with $\mathrm{x} 4, \mathrm{x} 8, \mathrm{x} 16$, and $\mathrm{x} 32$. The results show that TEES can significantly reduce the temperature of active disks, even with the accelerated trace replay speed. Based on our comprehensive experiments, TEES reduces the temperature by $9.12{ }^{\circ} \mathrm{C}$ compared to Rabbit.

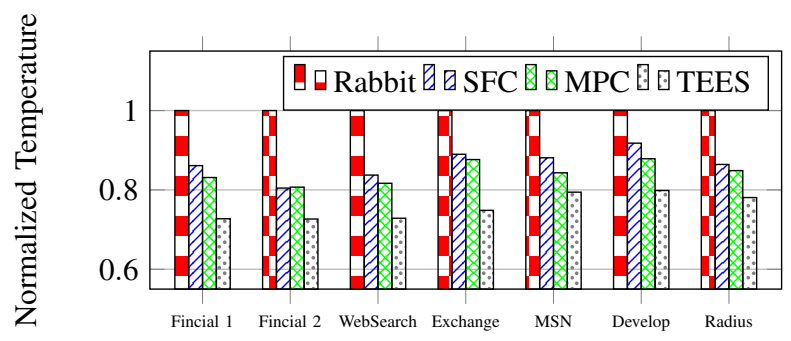

Fig. 10. Normalized Temperature under Different Traces.

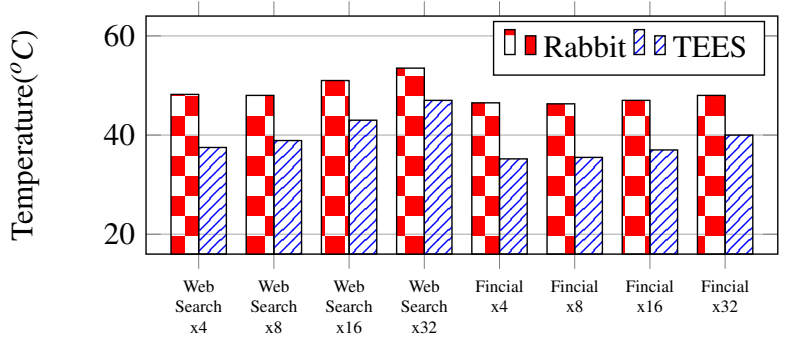

Fig. 11. Temperature with Accelerated Trace Replay.

5) Energy Consumption: In this experiment, we evaluate the energy consumption under different workloads. Figure 12 shows that TEES has a minimal impact on the energy consumption compared with normal power proportional storage. Figure 13 plots the power consumption of TEES and Rabbit with accelerated trace replay. The results show that we get almost the same power proportional property as Rabbit, with a mean absolute percentage error under $1 \%$. Note that for the WebSearch $\mathrm{x} 32$, the power consumption of TEES is much lower than Rabbit. That is because our fuzzy controller steps into a performance degraded model to achieve the best satisfaction of the control object. 


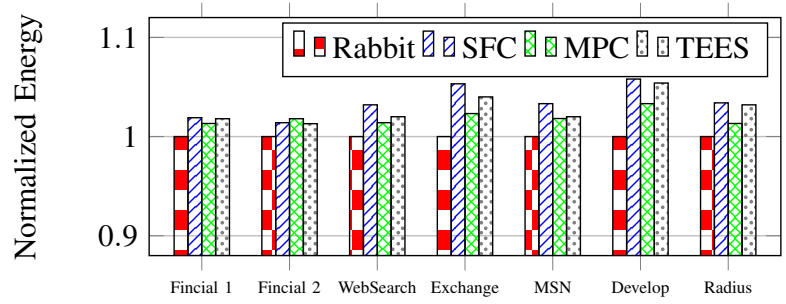

Fig. 12. Normalized Energy Consumption under Different Traces.

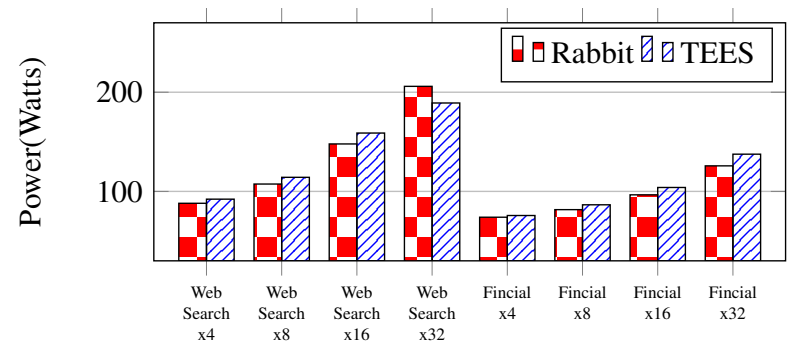

Fig. 13. Power Consumption with Accelerated Trace Replay.

6) Performance: In this experiment, we evaluate the impact of our controller on the performance at different I/O intensities. Figure 14 plots the average I/O delay under different workloads. It shows that TEES makes the least impact on the performance. This is because TEES has a very accurate prediction module and it can dynamically change the temperature threshold when the temperature vibrates. Figure 15 plots the average IOPS of TEES and Rabbit with accelerated trace replay. The result shows that we get the same I/O performance with Rabbit, with a mean absolute percentage error under $1 \%$. Note that for the WebSearch $\mathrm{x} 32$, the I/O performance of TEES is much lower than Rabbit. That is because TEES estimator calculated that the temperature would surpass $50{ }^{\circ} \mathrm{C}$. Therefore, TEES steps into a performance degree model. Figure 16 plots the IOPS tracking of Rabbit, SFC, and TEES respectively. SFC has a great performance degradation whenever it takes the control actions, while the TEES has little degradation. We can see that TEES obtains a faster and more accurate tracking behavior than SFC.

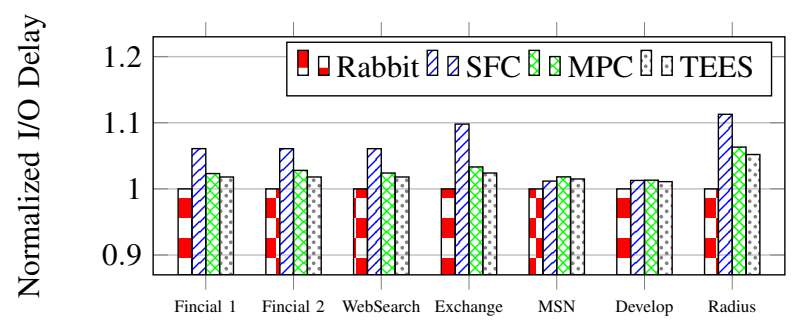

Fig. 14. Normalized I/O Delay under Different Traces.

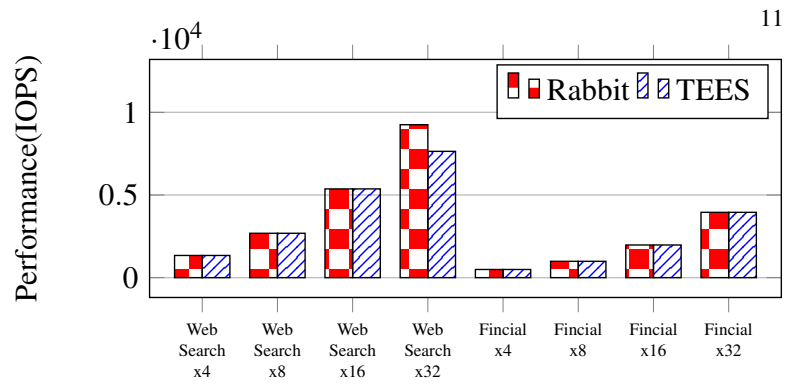

Fig. 15. IOPS with Accelerated Trace Replay.

\section{Simulation}

In this subsection, we use simulations to demonstrate the scalability of TEES when the number of disks increases to 32,64 , and 128 . We extract the power, temperature and performance model from previous tests and use event-driven simulation strategies. We read the percentage of the aggregated satisfaction from the simulator. As shown in Figure 17, TEES generates higher satisfaction degrees when the scale is larger, because it is easier to find candidate low-temperature disks.

\section{Conclusions}

Energy consumption has become an important issue in storage systems. Existing energy control solutions emphasize power consumption without considering reliability degradation that results from overburden of those long standing disks. In this paper, we present the first study of applying a Fuzzy Decision Making theory in energy-efficient storage systems to manage the energy while adhering to the temperature constraints and maintaining the power proportional attributes of the storage systems. Comprehensive experimental results on a physical test-bed show that TEES outperforms two state-of-art algorithms by significantly reducing the temperature in energy-efficient storage systems. More specifically, TEES reduces the temperature by $20 \%$ $30 \%$ compared to current methods such as Rabbit and MPC, while maintaining comparable performance and power consumption.

\section{Acknowledgements}

This work is supported in part by the US National Science Foundation Grant CCF-1527249, CCF-1337244, US National Science Foundation Early Career Award 0953946, PRC National Science Foundation Grant No. 61300047, 61572209 and National Natural Science Foundation of Hubei Province No.2015CFB315. 


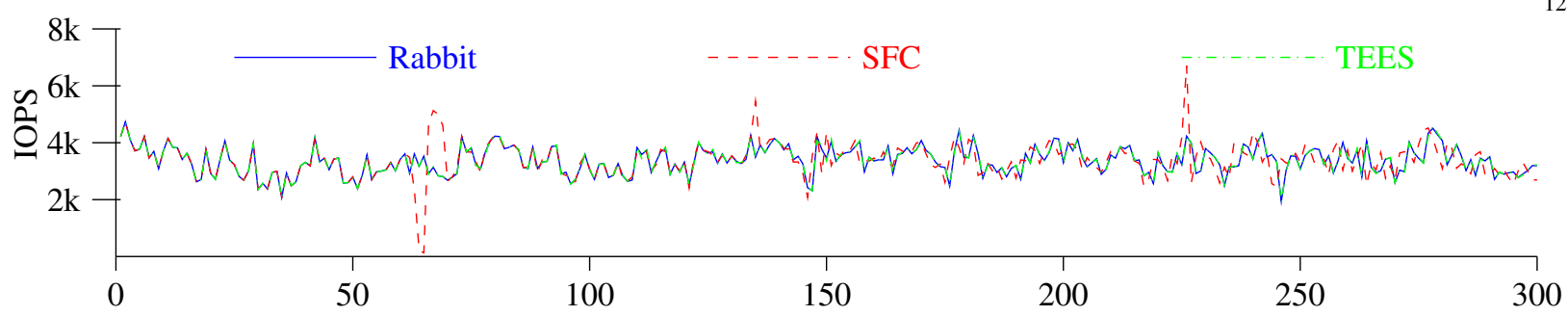

Time(minutes)

Fig. 16. Performance Tracking. TESS has the same performance compared to original Rabbit, while the simple feedback control create a noticeable I/O delay while switch the active disk set.

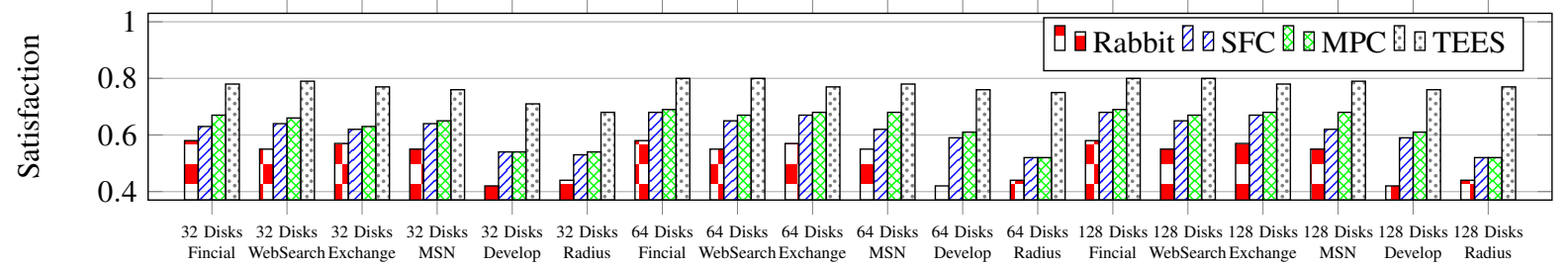

Fig. 17. Large-scale Simulation. We use static control parameters learned from real world tests to simulate the large-scale activities. We use satisfaction degree to indicate the overall control results.

\section{References}

[1] Rich Miller. Google: Raise your data center temperature. http://www.datacenterknowledge.com/archives/2008/10/ 14/google-raise-your-data-center-temperature/, 2008.

[2] Nosayba El-Sayed, Ioan A Stefanovici, George Amvrosiadis, Andy A Hwang, and Bianca Schroeder. Temperature management in data centers: Why some (might) like it hot. ACM SIGMETRICS Performance Evaluation Review, 40(1):163-174, 2012.

[3] Shu Yin, Kenli Li, Xiao Qin, Xiaojun Ruan, Haiquan Chen, Jiong Xie, and Xiaomin Zhu. Reliability analysis of energyefficient parallel storage systems. In High Performance Computing and Communications \& 2013 IEEE International Conference on Embedded and Ubiquitous Computing (HPCC_EUC), 2013 IEEE 10th International Conference on, pages 1448-1455. IEEE, 2013.

[4] Y. Wang, K. Ma, and X. Wang. Temperature-constrained power control for chip multiprocessors with online model estimation. In ACM SIGARCH Computer Architecture News, volume 37, pages 314-324. ACM, 2009.

[5] X. Wang and M. Chen. Cluster-level feedback power control for performance optimization. In High Performance Computer Architecture, 2008. HPCA 2008. IEEE 14th International Symposium on, pages 101-110. IEEE, 2008.

[6] G. Chen, W. He, J. Liu, S. Nath, L. Rigas, L. Xiao, and F. Zhao. Energy-aware server provisioning and load dispatching for connection-intensive internet services. In Proceedings of the 5th USENIX Symposium on Networked Systems Design and Implementation, volume 8, pages 337-350. USENIX Association, 2008.

[7] J. Moore, J. Chase, P. Ranganathan, and R. Sharma. Making scheduling" cool": temperature-aware workload placement in data centers. In Proceedings of the annual conference on USENIX Annual Technical Conference, pages 5-5, 2005.

[8] C. Bash and G. Forman. Cool job allocation: measuring the power savings of placing jobs at cooling-efficient locations in the data center. In 2007 USENIX Annual Technical Conference on Proceedings of the USENIX Annual Technical Conference, page 29. USENIX Association, 2007.

[9] Eduardo F Camacho, Carlos Bordons, Eduardo F Camacho, and Carlos Bordons. Model predictive control, volume 2. Springer London, 2004

[10] J. Wang, H. Zhu, and D. Li. eraid: Conserving energy in conventional disk-based raid system. Computers, IEEE Transactions on, 57(3):359-374, 2008.

[11] E. Pinheiro and R. Bianchini. Energy conservation techniques for disk array-based servers. In Proceedings of the 18th annual international conference on Supercomputing, pages 6878, 2004.

[12] Q. Zhu and Y. Zhou. Power-aware storage cache management. Computers, IEEE Transactions on, 54(5):587-602, 2005.

[13] D. Colarelli and D. Grunwald. Massive arrays of idle disks for storage archives. In Proceedings of the 2002 ACM/IEEE conference on Supercomputing, pages 1-11. IEEE Computer Society Press, 2002.

[14] L. Useche, J. Guerra, M. Bhadkamkar, M. Alarcon, and R. Rangaswami. Exces: External caching in energy saving storage systems. In High Performance Computer Architecture, 2008. HPCA 2008. IEEE 14th International Symposium on, pages 89100. IEEE, 2008.

[15] R.T. Kaushik and M. Bhandarkar. Greenhdfs: Towards an energy-conserving storage-efficient, hybrid hadoop compute cluster. In Proceedings of the USENIX Annual Technical Conference, 2010.

[16] A. Verma, R. Koller, L. Useche, and R. Rangaswami. Srcmap: Energy proportional storage using dynamic consolidation. In Proceedings of the 8th USENIX Conference on File and Storage Technologies, pages 20-20. USENIX Association, 2010.

[17] Fangming Liu, Zhi Zhou, Hai Jin, Bo Li, Baochun Li, and Hongbo Jiang. On arbitrating the power-performance tradeoff in saas clouds. Parallel and Distributed Systems, IEEE Transactions on, 25(10):2648-2658, 2014.

[18] Ming Ma and Yuanyuan Yang. Sencar: an energy-efficient data gathering mechanism for large-scale multihop sensor networks. Parallel and Distributed Systems, IEEE Transactions on, 18(10):1476-1488, 2007. 
[19] C. Weddle, M. Oldham, J. Qian, A.I.A. Wang, P. Reiher, and G. Kuenning. Paraid: A gear-shifting power-aware raid. ACM Transactions on Storage (TOS), 3(3):13, 2007.

[20] D. Li and J. Wang. Eeraid: energy efficient redundant and inexpensive disk array. In Proceedings of the 11th workshop on ACM SIGOPS European workshop, page 29. ACM, 2004.

[21] B. Mao, D. Feng, H. Jiang, S. Wu, J. Chen, and L. Zeng. Graid: A green raid storage architecture with improved energy efficiency and reliability. In Modeling, Analysis and Simulation of Computers and Telecommunication Systems, 2008. MASCOTS 2008. IEEE International Symposium on, pages 1-8. IEEE, 2008.

[22] H. Amur, J. Cipar, V. Gupta, G.R. Ganger, M.A. Kozuch, and K. Schwan. Robust and flexible power-proportional storage. In Proceedings of the 1st ACM symposium on Cloud computing, pages 217-228. ACM, 2010.

[23] Eduardo Pinheiro, Wolf-Dietrich Weber, and Luiz André Barroso. Failure trends in a large disk drive population. In FAST, volume 7, pages 17-23, 2007.

[24] S. Sankar, M. Shaw, and K. Vaid. Impact of temperature on hard disk drive reliability in large datacenters. In Dependable Systems \& Networks (DSN), 2011 IEEE/IFIP 41st International Conference on, pages 530-537. IEEE, 2011.

[25] Sriram Sankar, Mark Shaw, Kushagra Vaid, and Sudhanva Gurumurthi. Datacenter scale evaluation of the impact of temperature on hard disk drive failures. Trans. Storage, 9(2):6:1-6:24, July 2013.

[26] Western Digital. Thermal reliability technical paper.

[27] D. Narayanan, A. Donnelly, and A. Rowstron. Write offloading: Practical power management for enterprise storage. ACM Transactions on Storage (TOS), 4(3):10, 2008.

[28] Xu Yang, Zhou Zhou, Sean Wallace, Zhiling Lan, Wei Tang, Susan Coghlan, and Michael E Papka. Integrating dynamic pricing of electricity into energy aware scheduling for hpc systems. In Proceedings of the International Conference on High Performance Computing, Networking, Storage and Analysis, page 60. ACM, 2013.

[29] T. Heath, A.P. Centeno, P. George, L. Ramos, Y. Jaluria, and R. Bianchini. Mercury and freon: temperature emulation and management for server systems. In ACM SIGARCH Computer Architecture News, volume 34, pages 106-116. ACM, 2006.

[30] Z. Abbasi, G. Varsamopoulos, and S.K.S. Gupta. Thermal aware server provisioning and workload distribution for internet data centers. In Proceedings of the 19th ACM International Symposium on High Performance Distributed Computing, pages 130-141. ACM, 2010.

[31] A. Weissel and F. Bellosa. Dynamic thermal management for distributed systems. In Proceedings of the 1st Workshop on Temperature-Aware Computer Systems, 2004.

[32] L. Ramos and R. Bianchini. C-oracle: Predictive thermal management for data centers. In High Performance Computer Architecture, 2008. HPCA 2008. IEEE 14th International Symposium on, pages 111-122. IEEE, 2008.

[33] João Miguel da Costa Sousa and Uzay Kaymak. Model predictive control using fuzzy decision functions. Systems, Man, and Cybernetics, Part B: Cybernetics, IEEE Transactions on, 31(1):54-65, 2001.

[34] Zsofi Ruttkay. Fuzzy constraint satisfaction. In Fuzzy Systems, 1994. IEEE World Congress on Computational Intelligence., Proceedings of the Third IEEE Conference on, pages 12631268. IEEE, 1994.

[35] Ronald R Yager. On ordered weighted averaging aggregation operators in multicriteria decisionmaking. Systems, Man and Cybernetics, IEEE Transactions on, 18(1):183-190, 1988.

[36] I/o. umass trace repository. http://traces.cs.umass.edu/index.php/ Storage/Storage.

[37] Microsoft enterprise traces. http://iotta.snia.org/tracetypes/3. 